\title{
Institutional Members
}

\section{Corporate Members}

\section{Agfa Healthcare}

Mortsel, Belgium

www.agfa.com

American Health Information Management Association (AHIMA)

Chicago, IL - USA

www.ahima.org

Cerner Corporation

Kansasa City, MO - USA

www.cerner.com

Elsevier Science, Health Sciences Division

London - UK

www.elsevierhealth.com/journals/ijmi

Healthcare Information \& Management Systems Society (HIMSS)

Chicago, IL - USA

www.himss.org

IBM Corporation

Hawthorne, NY - USA

www.ibm.com/us

Ormed Information Systems Ltd.

Edmonton, AB - Canada

www.ormed.com

Saudi German Hospitals Group (Provisional)

Jeddah Al Zahraa - Saudi Arabia

www.sghgroup.com

Schattauer GmbH

Stuttgart - Germany

www.schattauer.com

Sensis Corporation

East Syracuse, NY - USA

Www.sensis.com

Siemens Medical Solutions

Erlangen - Germany

www.siemensmedical.com

\section{Academic Members}

\author{
Aalborg University \\ Aalborg - Denmark \\ www.v-chi.dk \\ Academic Medical Center (AMC) \\ Amsterdam - The Netherlands \\ kikpage.amc.uva.nl
}

\begin{abstract}
Central Queensland University
Rockhampton, NSW - Australia

www.infocom.cqu.EDU.AU
\end{abstract}

Centre for Health Informatics, Research and Development (CHIRAD)

Winchester - United Kingdom

www.chirad.org.uk

Columbia University, Department of Biomedical Informatics New York, NY - USA

www.dbmi.columbia.edu

Commission on Accreditation for Health Informatics and Information Management Education (CAHIIM) of AHIMA

Chicago, IL - USA

www.ahima.org

ErasmusUniversity Medical Center

Rotterdam - The Netherlands

www.bmg.eur.nl/smw/eimhim

Erasmus University Rotterdam

Rotterdam - The Netherlands

www.eur.nl/fgg/mi

Galil Center for Telemedicine and Medical Informatics

Haifa - Israel

www.galilcenter.org.il

Georg-August-University Goettingen

Goettingen - Germany

www.med.uni-goettingen.de

Glasgow Caledonian University

Glasgow, Scotland - United Kingdom

http://www.gcal.ac.uk

Harvard Medical School

Boston, MA - USA

Www.mi-boston.org/fellowship

IMGT, the international ImMunoGeneTics information system ${ }^{\circledR}$

Montpellier, France

imgt.cines.fr

Information Technology Institute (ITI) (Provisional)

Giza - Egypt

www.iti.gov.eg

Johns Hopkins University

Laurel MD - USA

www.jhuapl.edu

King Saud bin Abdulaziz University for Health Sciences (KSAU-HS)

Riyadh, Kingdom of Saudi Arabia

www.ksau-hs.edu.sa 
King Saud University

Riyadh - Kingdom of Saudi Arabia

www.ksu.edu.sa

National and Kapodistrian University of Athens

Athens - Greece

www.nurs.uoa.gr

National Institute of Public Administration - Instituto

Nacional de Administração

Oeiras, Portugal

www.ina.pt

National Library of Medicine, Lister Hill National Center

for Biomedical Communications

Bethesda, MD - USA

www.lhncbc.nlm.nih.gov

NHS Connecting for Health

Birmingham- United Kingdom

www.nhsia.nhs.uk

Nova Southeastern University (Provisional)

Ft. Lauderdale Florida - USA

medicine.nova.edu/msmi

Oregon Health and Science University

Portland, OR - USA

www.ohsu.edu/dmice

Politecnico di Milano

Milan - Italy

www.biomed.polimi.it

Russian Academy of Medical Sciences (Provisional)

Moscow - Russia

www.mcramn.ru/indexe.htm

St George's - University of London

London - United Kingdom

www.pcdq.org

Stanford University

Stanford, CA - USA

www.smi.stanford.edu

Taiwan Association for Medical Informatics

Taipei - Taiwan

www.medinfo.org.tw

Technical University of Braunschweig

Braunschweig - Germany

www.tu-braunschweig.de/mi

University College Dublin

Dublin - Ireland

www.ucd.ie/medicine/healthinf

University College London (UCL)

London - United Kingdom

www.chime.ucl.ac.uk

University Department of Rural Health, Tasmania

Launceston, Tasmania - Australia

www.ruralhealth.utas.edu.au/informatics/
University for Health Informatics and Technology Tyrol (UMIT)

Innsbruck, Austria

www.umit.at

University of Alabama at Birmingham

Birmingham, AL - USA

www.uab.edu/hi

University of Central Lancashire

Preston Lancashire - United Kingdom

www.uclan.ac.uk

University of Heidelberg

Heidelberg - Germany

www.med.uni-heidelberg.de/mi

University of Kuopio (Provisional)

Kuopio - Finland

www.uku.fi/tht/english

University of Maryland (Baltimore), School of Nursing

Baltimore, MD - USA

nursing.umaryland.edu/users/eahpi/web/index.htm

University of Missouri-Columbia

Columbia, MO - USA

informatics.missouri.edu

University of New Mexico

Albuquerque NM - USA

hsc.unm.edu/library

University of Nottingham

Nottingham, UK

www.primis.nhs.uk

University of Sydney

Lidcombe, NSW - Australia

www.fhs.usyd.edu.au/him

University of Texas

Houston, TX - USA

www.shis.uth.tmc.edu

University of Tromsø (Provisional)

Tromsø - Norway

uit.no/informatikk/

University of Utah

Salt Lake City, UT - USA

University of Victoria

Victoria, BC - Canada

hinf.uvic.ca/

University of Washington

Seattle, WA - USA

www.bhi.washington.edu

University of Waterloo

Waterloo, ON - Canada

hi.uwaterloo.ca

University of Wollongong

Wollongong, NSW - Australia

www.uow.edu.au 